\title{
PhenX: Environment measures for Tobacco Regulatory Research
}

\author{
Jennifer B Unger (1) , ${ }^{1}$ Frank J Chaloupka, ${ }^{2}$ Donna Vallone, ${ }^{3}$ James F Thrasher, ${ }^{4}$ \\ Destiney S Nettles, ${ }^{5}$ Tabitha P Hendershot ${ }_{1}^{5}$ Gary E Swan, ${ }^{6}$ PhenX TRR Environment \\ Working Group
}

${ }^{1}$ Department of Preventive Medicine, University of

Southern California Keck School of Medicine, Los Angeles,

California, USA

${ }^{2}$ Department of Health Policy and Administration and Health Policy Center, University of Illinois at Chicago, Chicago, Illinois, USA

BDepartment of Research and Evaluation, Truth Initiative, Washington, Washington, DC, USA

${ }^{4}$ Arnold School of Public Health, University of South Carolina, Columbia, South Carolina, USA ${ }^{5}$ RTI International, Research Triangle Park, North Carolina, USA

${ }^{6}$ Stanford Prevention Research Center, Stanford University School of Medicine, Stanford California, USA

\section{Correspondence to}

Dr Jennifer B Unger, Department of Preventive Medicine, University of Southern California Keck School of Medicine, Los Angeles, CA 90089, USA; unger@usc.edu

Received 25 April 2018

Revised 16 June 2018

Accepted 19 June 2018

\section{Check for updates}

(C) Author(s) (or their employer(s)) [year]. No commercial re-use. See rights and permissions. Published by BMJ

To cite: Unger JB,

Chaloupka FJ, Vallone D,

et al. Tob Control

2020:29:s35-s42.

\section{ABSTRACT}

Objective A Working Group (WG) of tobacco regulatory science experts identified measures for the tobacco environment domain.

Methods This article describes the methods by which measures were identified, selected, approved and placed in the PhenX Toolkit.

Findings The WG identified 20 initial elements relevant to tobacco regulatory science and determined whether they were already in the PhenX Toolkit or whether novel or improved measures existed. In addition to the 10 complementary measures already in the Toolkit, the WG recommended 13 additional measures: aided and confirmed awareness of televised antitobacco advertising, interpersonal communication about tobacco advertising, media use, perceived effectiveness of antitobacco advertising, exposure to smoking on television and in the movies, social norms about tobacco (for adults and for youth), worksite policies, youth cigarette purchase behaviours and experiences, compliance with cigarette packaging and labelling policies, local and state tobacco control public policies, and neighbourhood-level racial/ethnic composition. Supplemental measures included youth social capital and compliance with smoke-free air laws and with point of sale and internet tobacco marketing restrictions. Gaps were identified in the areas of policy environment (public and private), communications environment, community environment and social environment (ie, the norms/ acceptability of tobacco use).

Conclusions Consistent use of these tobacco environment measures will enhance rigor and reproducability of tobacco research.

\section{INTRODUCTION}

The growing field of tobacco regulatory science depends on the use of valid measures that can be harmonised across studies. To obtain consensus about specific measures relevant to this field, the US Food and Drug Administration's (FDA) Center for Tobacco Products (CTP) and the National Institutes of Health (NIH) Tobacco Regulatory Science Program funded an expansion of the PhenX Toolkit to include one core collection of Tobacco Regulatory Research (TRR) measures and five specialty collections.

The PhenX TRR Panel (TRRP) identified Working Groups (WGs) using the categories in the 'Host, Agent, Vector and Environment (HAVE) model', an epidemiological framework that examines the HAVE model to understand the patterns, causes and effects of health and disease conditions. ${ }^{1}$ In the HAVE model, the Host denotes the consumers or potential consumers of tobacco products, the Agent is the tobacco products, the Vector is the tobacco industry and other entities that distribute and promote tobacco products, and the Environment is the social and physical context in which the Host, Agent and Vector operate. The Host category was subdivided into Social/Cognitive and Biobehavioral measures, resulting in the following five specialty areas: Host-Social/Cognitive, Host-Biobehavioral, Agent, Vector and Environment. This article describes the selection of the environment measures.

The Environment WG was assembled to identify measures of environmental factors that influence tobacco product use. In the original HAVE model, ${ }^{1}$ the Environment includes 'social, cultural, historical, political, economic, media, public health and legal factors'. Initial discussions about the content of the HAVE areas revealed that some constructs could potentially fit into both Vector and Environment. For example, tobacco marketing is a component of Vector when the tobacco industry disseminates tobacco advertisements, but it becomes part of the Environment when tobacco users and potential users encounter those advertisements in the community. After suggestions to combine the two WGs, the TRRP decided to keep them separate because the scope of each WG was so broad. This led to a decision to place measures of youth access and exposure to smoking in entertainment media into the Environment WG. The TRRP decided that tobacco products and messages about tobacco products are part of the Vector as they emanate from the tobacco industry, and then they become part of the Environment when users and potential users encounter and perceive them.

Previous work in this area was conducted by the Environment WG of the National Tobacco Monitoring, Research and Evaluation Workshop sponsored by the National Cancer Institute and the American Legacy Foundation (now known as the Truth Initiative) in 2002. This group made recommendations to enhance the existing surveillance and evaluation systems in the context of a potential national Tobacco Surveillance Network. ${ }^{12}$ A major conclusion was that existing systems provided complete and timely data on state-level tobacco control policies but not sufficient detail on locallevel policies. This lack of information about local policies made it difficult to estimate the effects of policies on tobacco use outcomes. Thus, a gap identified at that time was measures of local tobacco 
Table 1 Initial and final environment scope

\begin{tabular}{|c|c|}
\hline Initial scope & Final scope \\
\hline 1. Third-party policies & 1. Policy environment \\
\hline 2. Business improvement district & a. Public \\
\hline 3. Clean indoor air policies & b. Private \\
\hline 4. Community disorganisation & 2. Communications environment \\
\hline 5. Coverage of smoking laws/policies & 3. Community environment \\
\hline 6. Cultural and community factors & 4. Social norms/acceptability \\
\hline 7. Expenditures on tobacco prevention & \\
\hline 8. Exposure to antitobacco campaigns & \\
\hline 9. Institutional setting & \\
\hline 10. Local tobacco use prevalence & \\
\hline 11. Neighbourhood poverty & \\
\hline 12. Population density & \\
\hline 13. Retail outlet density and proximity & \\
\hline $\begin{array}{l}\text { 14. Secondhand smoke in the physical } \\
\text { environment }\end{array}$ & \\
\hline 15. Smoke-free environments & \\
\hline 16. Smoking bans & \\
\hline 17. Taxes/price & \\
\hline 18. Smokers in environment & \\
\hline 19. Minors access & \\
\hline $\begin{array}{l}\text { 20. Exposure to smoking in the } \\
\text { entertainment media/product } \\
\text { placement }\end{array}$ & \\
\hline
\end{tabular}

control laws/ordinances and tobacco programme activities. Other high-priority gaps included the need for better monitoring of cigarette taxes/prices and tobacco counter marketing. The charge of the Environment WG was to fill these gaps and identify new and improved measures of the environment relevant to tobacco regulatory science.

\section{METHODS}

\section{Expertise of the WG}

Environment WG members were selected based on their expertise in the development, evaluation and use of environment measures, including assessing the effects of counter-marketing campaigns, state and local tobacco control policies, compliance with smoke-free air laws and tobacco product restrictions, and tobacco-related norms, perceptions and behaviours. The WG consisted of two cochairs, four content experts and two TRRP liaisons. Environment specialty area experts from the FDA and NIH also participated. In addition, the WG members consulted with numerous specialty area experts regarding the availability and selection of specific protocols.

\section{Selection of thematic areas}

The initial scope of the Environment WG was selected by the TRRP. From this broad set, the Environment WG members developed four broad thematic areas (table 1).

\section{Complementary measures already in the PhenX Toolkit}

The PhenX Toolkit requires that measures cannot compete with one another. At the time the Environment WG was convened, over 366 measures were in the Toolkit. To avoid overlap between existing and new recommended measures, WG members identified existing measures related to environmental constructs. Ten existing PhenX measures were deemed complementary to the Environment scope: three from the substance abuse and addiction specialty collection, one from the respiratory domain, one from the cancer domain, one from the environmental exposures domain and four from the social environments domain (table 2).

\begin{tabular}{lll}
\hline Table 2 & Complementary environment measures in the PhenX Toolkit \\
\hline Subgroup & Complementary PhenX measures & $\begin{array}{l}\text { PhenX domain/ } \\
\text { specialty collection }\end{array}$ \\
\hline $\begin{array}{l}\text { Policy } \\
\text { environment }\end{array}$ & $\begin{array}{l}\text { Point-of-sale environment for alcohol } \\
\text { and tobacco (PhenX id=550801) } \\
\text { Cigarette price (PhenX id=550101) }\end{array}$ & $\begin{array}{l}\text { SAA (specialty } \\
\text { collection) }\end{array}$ \\
& $\begin{array}{l}\text { Urine assay for tobacco smoke exposure } \\
\text { (PhenX id=091701) }\end{array}$ & Respiratory (domain) \\
& $\begin{array}{l}\text { Passive smoke exposure (PhenX } \\
\text { id=070301) }\end{array}$ & Cancer (domain) \\
& $\begin{array}{l}\text { Current environmental tobacco smoke } \\
\text { exposure (PhenX id=060701) }\end{array}$ & $\begin{array}{l}\text { Environmental } \\
\text { exposures (domain) }\end{array}$ \\
Community & $\begin{array}{l}\text { Neighbourhood safety (PhenX } \\
\text { id=210901) }\end{array}$ & $\begin{array}{l}\text { Social environments } \\
\text { (domain) }\end{array}$ \\
& $\begin{array}{l}\text { Neighbourhood collective efficacy- } \\
\text { environment }\end{array}$ & \\
& $\begin{array}{l}\text { community cohesion and informal social (PhenX id=210801) } \\
\text { Neighbourhood concentrated }\end{array}$ & \\
& $\begin{array}{l}\text { disadvantage (PhenX id=211302) } \\
\text { Race/ethnic residential segregation } \\
\text { (PhenX id=211400) }\end{array}$ & \\
& $\begin{array}{l}\text { Community risk and protective factors } \\
\text { (PhenX id=540101) }\end{array}$ & $\begin{array}{l}\text { SAA (specialty } \\
\text { collection) }\end{array}$ \\
& &
\end{tabular}

Communications environment and social norms/acceptability have no complementary measures in the Toolkit.

SAA, substance abuse and addiction.

PhenX staff also provided information about potentially competing measures proposed by other TRR WGs. In reviewing the measures suggested by the Vector WG, the Environment WG provided input regarding their proposed protocol for exposure to tobacco marketing through internet and social media use. The Environment WG recommended that questions from the Truth Longitudinal Cohort (TLC) study be included as the questions provide specificity in addition to frequency by asking the respondent to estimate time spent watching television; time spent on a computer, smartphone or tablet and time spent on social media sites. This revision required the inclusion of three source protocols, but the PhenX criteria allow only two source protocols per construct. To resolve these issues, the TRRP proposed revising the current Vector measure related to general media use to include the items from the TLC protocol. Revising this measure could benefit all Toolkit users across different domains, in addition to those interested in tobacco marketing exposure.

\section{WG deliberations}

The Environment WG met for an introductory call on 19 November 2014 and then assembled in person on 23 January 2015. The WG engaged in thoughtful discussions to decide which measures should be included in the Toolkit. Priority was given to measures assessing exposure, perceptions and engagement with both marketing and counter marketing; compliance with federal, state and local tobacco product marketing policies and smoke-free air laws; and how the social environment may influence tobacco product use. Measures also were prioritised based on their relevance to multiple tobacco products now under the regulatory purview of the FDA: cigarettes, roll-your-own tobacco and smokeless tobacco, which have been regulated by the FDA since 2009; and newly deemed products made or derived from tobacco, such as electronic cigarettes (e-cigarettes), pipe tobacco, hookah tobacco, cigars and future products, which were being considered for FDA regulation at the time of the meeting and have been regulated by the FDA since August 
Table 3 Final environment measures

\begin{tabular}{|c|c|}
\hline Measure & Description of measurement protocol \\
\hline $\begin{array}{l}\text { 1. Aided and confirmed awareness of televised antitobacco advertising (PhenX } \\
\text { id=750101) }\end{array}$ & $\begin{array}{l}\text { Self-administered or interviewer-administered protocol assesses whether a respondent has seen any } \\
\text { televised antitobacco/antismoking advertisements, and if so, the respondent is asked to describe the } \\
\text { advertisement. }\end{array}$ \\
\hline 2. Compliance with cigarette packaging and labelling policies (PhenX id=750201) & $\begin{array}{l}\text { Observational protocol assesses cigarette pack identifiers or product codes, general pack characteristics, } \\
\text { tax stamps, promotions and counterfeit packs. }\end{array}$ \\
\hline 3. Interpersonal communication about antitobacco advertising (PhenX id=750501) & $\begin{array}{l}\text { Self-administered or interviewer-administered protocol assesses whether respondents have discussed } \\
\text { antitobacco advertisements with anyone, and if so, follow-up items assess receptivity to the message. }\end{array}$ \\
\hline 4. Local tobacco control public policies (PhenX id=750601) & $\begin{array}{l}\text { Secondary data analysis protocol assesses local ordinances and board of health regulations containing } \\
\text { provisions on clean indoor air, restrictions on youth access to tobacco and other policies. }\end{array}$ \\
\hline 5. Media use (PhenX id=750701) & $\begin{array}{l}\text { Self-report protocol assesses amount of time spent using different media tools and viewing various social } \\
\text { media sites. }\end{array}$ \\
\hline 6. Neighbourhood-level racial/ethnic composition (PhenX id=750801) & $\begin{array}{l}\text { Secondary data analysis protocol uses data from the US Census Bureau on the racial/ethnic composition of } \\
\text { neighbourhoods, which can be calculated at the census tract or block group level. }\end{array}$ \\
\hline 7. Perceived effectiveness of antitobacco advertising (PhenX id=750901) & $\begin{array}{l}\text { Self-report protocol assesses the perceived effectiveness of antitobacco advertisements in relation to } \\
\text { tobacco-related behaviours and outcomes. }\end{array}$ \\
\hline $\begin{array}{l}\text { 8. Self-reported exposure to smoking on television and in the movies (PhenX } \\
\text { id=751001) }\end{array}$ & Protocol assesses the perceived exposure to people who smoke in movies or on television. \\
\hline 9. Social norms about tobacco & $\begin{array}{l}\text { Adult: Interviewer-administered protocol assesses social norms within the adult population (PhenX } \\
\text { id=750301). }\end{array}$ \\
\hline 10. & $\begin{array}{l}\text { Youth: Self-report or interviewer-administered protocol assesses descriptive norms (ie, others' use of } \\
\text { tobacco) and injunctive norms (ie, others' approval of tobacco use) within the youth population (PhenX } \\
\text { id=750302) }\end{array}$ \\
\hline 11. State tobacco control public policies (PhenX id=751200) & $\begin{array}{l}\text { Secondary data analysis protocol assesses current and historical state-level data on tobacco use } \\
\text { prevention and control laws and policies. }\end{array}$ \\
\hline 12. Worksite policies (PhenX id=751401) & $\begin{array}{l}\text { Interviewer-administered protocol assesses a respondent's exposure to and compliance with worksite } \\
\text { policies on tobacco. }\end{array}$ \\
\hline 13. Youth cigarette purchase behaviours and experiences (PhenX id=751501) & Self-administered protocol assesses youth purchase behaviours and experiences. \\
\hline $\begin{array}{l}\text { Additional measures included in the Outreach process but not selected for inclusion in } \\
\text { Compliance with Smoke-free Air Laws. } \\
\text { Compliance with Tobacco Marketing Restrictions. } \\
\text { Support for Tobacco-related Policies. } \\
\text { Youth Cigarette Purchase Behaviours and Experiences. }\end{array}$ & PhenX \\
\hline
\end{tabular}

2016. The WG reviewed a comprehensive list of environment measures, including self-reported, observational and secondary data elements.

\section{Community Outreach results}

Community Outreach was implemented 18 May 2015 to 29 May 2015. The measures included in the Community Outreach process are shown in table 3 (all 13 final measures plus the 4 additional measures at the bottom of table 3). The Outreach process elicited 13 responses. The reviewers suggested that the measures could be used for additional tobacco products not mentioned in the protocols. The WG agreed to provide some general guidance for these measures to indicate when the WG felt that a particular protocol could be used to elicit information about other tobacco products. For the measure compliance with tobacco marketing restrictions at point of sale, feedback indicated that it was important to obtain both the type of store and price promotions.

\section{RESULTS}

The WG identified 13 measures for inclusion in the PhenX Toolkit and four measures for the supplemental information (SI). The SI includes protocols that the WG considered useful and relevant but that were lower priorities than those selected for the Toolkit. Within the area of environmental tobacco control policy, measures were selected to assess compliance with cigarette packaging and labelling policies, tobacco control public policies at the state and local levels, and worksite policies. With respect to the broad communication environment, the WG selected measures related to exposure, perceptions and engagement with both marketing and counter marketing and general media use.
WG members also selected two community-level environment measures: the neighbourhood-level racial/ethnic composition for the Toolkit and a self-reported questionnaire to capture youth social capital for the SI. Table 3 shows the final 12 measures (13 protocols), which are described in more detail below.

\section{Aided and confirmed awareness of televised antitobacco advertising (PhenX id $=750101$ )}

Mass media campaigns are a cost-effective feature of the tobacco control environment. ${ }^{34}$ Evaluating such campaigns can inform future campaign development and funding priorities. Many campaign evaluations assess confirmed (or unaided) advertisement awareness, whereby participants must remember details about the advertisement to be considered aware. ${ }^{5}$ This is generally considered to be a superior measure of advertising campaign or message awareness. However, when resources or the data collection modality do not allow assessing confirmed awareness, aided awareness is a reasonable and valid alternative. ${ }^{6-9}$ This protocol includes two questions from Sly et $a l^{10}$ and Niederdeppe. ${ }^{11}$ These questions assess whether respondents have seen any televised counter marketing advertisements; if so, they are asked to describe the advertisement. For aided awareness, respondents confirm whether they have seen the advertisement; for confirmed awareness, respondents provide additional details about the advertisement.

For aided awareness, items that provide a brief description or picture of an advertisement are based on the specific advertisements being assessed. The purpose is to provide just enough information to prompt accurate recall or recognition of the advertisement without showing it in its entirety (also called 'ad recognition'). ${ }^{5}$ Correct responses for question 2 are based on the 
specific advertisements being assessed. The WG acknowledges that these questions may also be used for advertisements aired online or through other media channels, such as radio or billboards. The protocol is intended for use in adults (18 years and older) and youth (12-17 years of age) and is available in English, although it has been adapted to Spanish. ${ }^{12}$ The questionnaire can be self-administered or interviewer administered.

\section{Compliance with cigarette packaging and labelling policies (PhenX id=750201)}

Tobacco product packaging is a key element of tobacco marketing. Tobacco product regulations have targeted tobacco product packaging, including limiting the use of various descriptors and requiring health warning labels. ${ }^{13}$ Packs that are not compliant with these regulations reflect either industry non-compliance or illicit products. This protocol is used to assess state and local government and retailer compliance with cigarette packaging and labelling policies.

The National Cancer Institute's study 'Maximising State and Local Policies to Restrict Tobacco Marketing at Point of Sale' uses the U01 Point of Sale: Pack Analysis Coding Training Manual (2013) to provide guidance on the collection, preservation and coding processes for cigarette packs purchased during store audits. A total of 49 items provide information on cigarette pack identifiers or product codes, general pack characteristics, tax stamps, promotions and counterfeit packs.

The WG recommended that this manual be used as a template. Investigators are advised to adapt the template to meet their specific research interests and needs. The template provided in the Toolkit focuses on Marlboro and Newport cigarette packs but could be used for other brands. This observational protocol requires the use of gloves and masks for handling tobacco products and was judged to be of 'high burden' (ie, taking longer than 15 min to complete). However, given its critical role in monitoring tobacco product sales-related policies, the WG recommended this measure. Given that the tobacco industry and its products evolve over time, standard language was included to provide guidance on clearly defining tobacco products. This measure is available in English.

\section{Interpersonal communication about antitobacco advertising (PhenX id=750501)}

Counter-marketing messages that prompt interpersonal discussions impact smoking decisions, ${ }^{8}{ }^{14-18}$ including cessation attempts. ${ }^{18} 19$ This protocol includes two questions from the Evaluation of the Public Education Campaign on Teen Tobacco questionnaire. ${ }^{20}$ Question 1 assesses whether respondents talked about a counter-marketing advertisement or media campaign with friends, family and/or others. If respondents have discussed the advertisement, they are asked follow-up items to determine their receptivity to the message. Next, they are asked specific questions related to the goals and objectives of the message; these secondary questions vary across messages. This protocol is typically administered after asking about confirmed and/or aided awareness of an antitobacco advertisement(s) (see the Aided and confirmed awareness of televised antitobacco advertising section).

Once respondents indicate recognition or recall of an advertisement, they should be asked whether they talked to anyone about the advertisement(s) (item \#1). If the respondent answers 'yes', they should be asked what topics were discussed (item \#2). The protocol can be administered via self-report-based or interviewer-based methodology. It is available in English and can be used in youth and adult samples, with adaptations to Spanish for assessing interpersonal communication about warning labels. ${ }^{19}$

Local tobacco control public policies (PhenX id $=750601$ )

Extensive evidence demonstrates the effectiveness of various tobacco control policies in reducing tobacco use..$^{21-25}$ In many jurisdictions, local tobacco control policies are stronger and/or more comprehensive than state policies. Therefore, accurately assessing local policies (in addition to state policies) is necessary to estimate associations between policies and tobacco use behaviour. $^{2}$

This secondary data analysis protocol uses tobacco control legislative data from the Americans for Non-smokers' Rights Foundation's US Tobacco Control Laws Database, a standardised system and national collection of local ordinances and board of health regulations containing provisions on clean indoor air, restrictions on youth access to tobacco, tobacco sales and distribution restrictions, tobacco advertising and promotion restrictions, tobacco excise taxes and conditional use permits (http:// www.no-smoke.org/goingsmokefree.php?id=519). Navigating the database requires the use of English, knowledge of the database, analytical expertise and a computer with internet access.

\section{Media use (PhenX id=750701)}

Media use provides opportunities to be exposed to protobacco and/or antitobacco messages. This self-report protocol uses two questions from the Truth Initiative's TLC Study (Truth Initiative, 2014) (items 510 and 520) to assess the average amount of time spent using different media tools and viewing various social media sites. The first item asks the respondent to provide the durations (hours and minutes) of watching television or other video sources; using a desktop or laptop to spend time online, playing games or watching videos and using a smartphone or tablet to use apps, play games or watch television. The second item assesses the amount of time per day spent on social media websites on a five-point scale ranging from 'none' to ' 6 or more hours'. The items are intended for use with both youth and adults and are available in both English and Spanish.

\section{Neighbourhood-level racial/ethnic composition (PhenX id $=750801$ )}

Measures of the racial/ethnic mix in the neighbourhood are relevant to tobacco regulatory science because tobacco use and social norms vary by race, ethnicity and acculturation ${ }^{26} 27$ and because tobacco retailers and tobacco advertising are more prevalent in specific racial/ethnic neighbourhoods. ${ }^{28}$ There is less exposure to US social norms and advertising in ethnic enclaves and more exposure to tobacco advertising and higher tobacco outlet density in minority neighbourhoods. ${ }^{29-34}$

This secondary data analysis protocol uses data from the US Census Bureau on the racial/ethnic composition of neighbourhoods, which can be calculated at the census tract or block group level. The relevant variables are available from the long form of the 2010 decennial Census (http:// www.census.gov/prod/ cen2010/doc/sf1.pdf). Depending on the focus of the study, the user can use tables downloaded from the US Census website (http://www.census.gov) to calculate the following variables: per cent white, black or African American, American Indian and Alaska Native, Asian, Native Hawaiian or Pacific Islander or some other race. Because people of every race can also be of Hispanic/Latino ethnicity, the user can refine these variables (eg, per cent non-Hispanic white, per cent Hispanic white, per cent non-Hispanic black or per cent Hispanic black). Census 
data also include the per cent foreign born and per cent who speak a language other than English to assess acculturation. The WG suggests that it is helpful for the user to have knowledge of Census data products and websites, such as American Factfinder, or commercial geospatial data products, such as those provided by vendors including GeoLytics (http://www.geolytics.com). Using the dataset requires facility with English, internet access to download raw data from the US Census Bureau's American Factfinder website (http://factfinder.census.gov) and knowledge of statistical packages (eg, SPSS or SAS) for data manipulation.

\section{Perceived effectiveness of antitobacco advertising (PhenX id $=750901$ )}

Antitobacco messages can influence behaviour when they are persuasive and memorable, evoke emotional reactions, and do not produce high levels of reactance; therefore, it is important to understand individuals' reactions to these messages. ${ }^{35}$ This protocol includes six questions from the Perceived Effectiveness of Cessation Advertisements study ${ }^{36}$ to assess whether the perceived effectiveness for antitobacco advertisements is causally related to tobacco-related behaviours and outcomes. Study participants are asked to provide their appraisals of advertising effectiveness after viewing an advertisement. The purpose of this measure is to evaluate respondents' reactions to antitobacco advertisements using a multi-item scale (worth remembering, grabbed attention, powerful, informative, meaningful and convincing; each rated on a five-point scale ranging from strongly disagree to strongly agree). Understanding the perceived effectiveness of specific types of antitobacco advertisements can help identify the message strategies that will be most effective for improving antitobacco-related behaviours and outcomes.

Each item reflects established characteristics of persuasive cessation messages, such as attention, recall, novelty, relevance and credibility. These measures can be used to compare cessation ads while also serving as proxy measures of campaign impact when it is not possible to assess longitudinal changes in campaign-targeted attitudes, beliefs and behaviours. The items are available in English, can be administered through self-reportbased or interviewer-based approaches, and are intended for use in adults.

\section{Self-reported exposure to smoking on television and in the movies (PhenX $i d=751001)$}

Exposure to smoking in movies promotes smoking initiation. ${ }^{37}$ WHO recommends policies to reduce youth exposure to tobacco imagery in entertainment media. ${ }^{38}{ }^{38}$ Fine-grained assessment of exposure to tobacco in films (eg, the Beach Method ${ }^{39}{ }^{40}$ ) requires significant resources and survey questions that are likely beyond the scope of most tobacco research. This protocol is taken from the Survey of Teen Opinions about Retail Environments, a school-based survey for adolescents. ${ }^{41}$ This item assesses frequency of perceived exposure to people who smoke in movies or on television in the past 7 days and provides four response options (never $=1$, often $=4$ ). The measure is intended for use in adolescents aged 11-14 years and is available in English.

\section{Social norms about tobacco}

Social norms are a key influence on behaviour ${ }^{42}$ because the rules and standards of a group guide or constrain social behaviour without the force of laws. ${ }^{43-45}$ Many tobacco control policies are presumed to reduce tobacco consumption by 'denormalising' tobacco use, making it socially unacceptable. ${ }^{46}$ Measures of social norms about tobacco tend to be either descriptive (ie, the prevalence of the behaviour among identity group members) or injunctive (ie, how identity group members believe one should behave). ${ }^{47} 48$ A literature review was conducted to determine the most frequently used measures in each of these domains and collect evidence of their reliability and validity. Studies of adults (18 years and older) and youth (aged $12-17$ years) mostly assessed perceptions of attitudes and behaviours related to tobacco product use among proximal network members, such as family and peers. The purpose of this measure is to evaluate tobacco-related attitudes and behaviours among important network members and the general population.

\section{Adult (PhenX id=750301)}

This protocol uses a combination of questions from the 2011 International Tobacco Control 4-Country Survey ${ }^{49}$ and the Population Assessment of Tobacco and Health (PATH) Study ${ }^{2050}$ instruments. The questions assess both descriptive and injunctive social norms. Assessments of descriptive norms include (1) whether a respondent resides with one or more users of any of six tobacco products and (2) the smoking status of the respondent's five closest friends or acquaintances. Injunctive norms are assessed by asking about perceived attitudes towards tobacco use by (1) most people and (2) the people who are most important to the respondent. These items are intended to be part of an interviewer-administered questionnaire and are available in Bengali, Dutch, English, French, German, Hindi, Irish, Korean, Malay, Portuguese, Spanish, Thai, Dzongkha and Maori.

\section{Youth (PhenX id=750302)}

This protocol includes one question from the wave 1 youth PATH Study, ${ }^{51}$ two questions from the wave 1 adult PATH Study ${ }^{50}$ and one question from the 'Changes in Peer and Parent Influence During Adolescence: Longitudinal versus CrossSectional Perspectives on Smoking Initiation' publication. ${ }^{52}$ As for adults, assessments of descriptive norms include both household members (ie, the respondent resides with one or more users of any of six tobacco products) and peers (ie, how many of the respondent's friends smoke). Additionally, injunctive norms (ie, others' approval of tobacco use) are queried for both close network members and, more generally, for most people. These items are intended to be part of an interviewer-administered questionnaire and are available in English and Spanish.

For question 1 in both the adult and youth protocols, the WG recommends that investigators provide descriptions and images of specific products while the questions are being administered. Furthermore, the WG recommends including e-cigarettes as a response option. Although questions 3 and 4 were extracted from the PATH Study adult questionnaire, the WG notes that they are also appropriate for youth. The WG noted that an additional, more objective indicator of descriptive norms could be constructed by determining the prevalence of tobacco use within a particular social group (eg, a school) when members of that group have provided self-report data about their own use. ${ }^{53}$ The youth protocol is intended for use in youth aged 12-17 years, is available in English and Spanish, and can be administered via self-report-based or interviewer-based formats.

\section{State tobacco control public policies (PhenX id $=751200$ )}

State public policy interventions and insurance mandates for cessation services through Medicaid are critical tools to prevent and reduce tobacco use by children, youth and adults. ${ }^{53-60}$ The protocol is based on extracting state legislative and Medicaid data from the CDC's State Tobacco Activities Tracking and Evaluation 
(STATE) system, an interactive data system that displays current and historical state-level data on tobacco use prevention and control laws and policies (https://www.cdc.gov/statesystem/ index.html). The STATE system integrates many data sources to provide comprehensive summary data and facilitate research and consistent data interpretation. Measures include comprehensive smoke-free air laws, pre-emption of local smoke-free air laws, excise taxes on tobacco products, restrictions on minors' access to tobacco products, marketing/advertising restrictions, policy requirements for Medicaid coverage for smoking cessation services and restrictions on emerging tobacco products. The STATE system provides instructions on how to generate reports, see state highlights and view interactive maps (https://www.cdc. gov/statesystem/index.html). Access to a computer with internet access, knowledge of the STATE system and the ability to analyse the data are requirements to use this resource.

\section{Worksite policies (PhenX id=751401)}

Policies against tobacco use in the workplace can increase cessation attempts and decrease exposure to secondhand smoke. ${ }^{61}{ }^{61}$ This protocol includes eight questions from the 2009 to 2010 National Adult Tobacco Survey (NATS) that assess a respondent's exposure to and compliance with worksite policies on tobacco. ${ }^{62}$ The $2009-2010$ NATS is a stratified national survey of US non-institutionalised adults aged 18 years and older that assesses the prevalence of tobacco use and the factors promoting and impeding tobacco use among adults. NATS establishes a comprehensive framework for evaluating both the national and state-specific tobacco control programmes. ${ }^{63}$ This protocol is intended for use in adults 18 years and older as an interviewer-administered questionnaire. It is available in English and Spanish. The interviewer must be trained to conduct personal interviews with individuals from the general population.

\section{Youth cigarette purchase behaviours and experiences (PhenX id=751501)}

This protocol includes five questions from the 2010 Monitoring the Future (MTF) project survey that capture data related to youth cigarette purchase behaviours and experiences. ${ }^{64}$ The MTF study is a reliable, valid, large-scale, national study that has been continuously administered to 12 th graders since 1975 . Since 1991,8 th and $10^{\text {th }}$ graders have also been included. ${ }^{65}$

This protocol is intended for use in youth aged 13-17 years as a self-administered questionnaire and is available in English. Items are focused on behaviours and experiences in the last 30 days, including method of acquisition, location of purchase, brand preference, experience with being asked for proof of age and whether the respondent has ever purchased just a few cigarettes in the past 12 months.

\section{Supplemental measures}

The three measures chosen for the SI included the Youth Social Capital measure (https://www.phenxtoolkit.org/toolkit_content/ supplemental_info/trsp_environment/measures/Youth_Social_ Capital.doc), which complements the existing adult measure of social capital in the Toolkit. This measure was recommended for the SI because the measure and protocol are not specific to tobacco regulation. The compliance with smokefree air laws (https://www.phenxtoolkit.org/toolkit_content/ supplemental_info/trsp_environment/measures/Compliance with_Smoke-free_Air_Laws.doc) and compliance with tobacco marketing restrictions (internet and point of sale) (https://www. phenxtoolkit.org/toolkit_content/supplemental_info/trsp_environment/measures/Compliance_with_Tobacco_Restrictions Internet.doc; https:/www.phenxtoolkit.org/toolkit_content/ supplemental_info/trsp_environment/measures/Compliance with Tobacco Restrictions POS.doc) were also recommende $\bar{d}$ for SI because the protocols for each are general and provide instruction on how to conduct a study instead of a specific method or set of questions to collect data.

\section{Measures not selected for inclusion}

As the primary goal of the TRR collections is to support research informing US federal regulatory activities, the measure, support for tobacco-related policies, was excluded as measures of public opinions of or support for policies is outside the scope of CTP. As such, this construct cannot be used to support the implementation of US federal tobacco regulations and guidelines. While other measures included in the environment collection assess policies that are also outside CTP's regulatory authority, including workplace-level, local-level and state-level policies related to smoke-free air laws and taxes, assessment of these policies was deemed helpful for research that comprehensively assesses the impact of factors or policies that could inform federal regulation.

\section{DISCUSSION}

To understand the role of environmental factors in tobacco product use, it is critical to use valid and comprehensive measures that incorporate changes in the portfolio of tobacco products, the communications landscape and the policy environment. The emergence of new tobacco products in the marketplace, new social norms about their use, new marketing strategies and health communication campaigns about these products, and new local-level, state-level and national-level policies about these products will necessitate periodic review and revision of these measures. The set of measures recommended by the Environment WG builds on previous recommendations ${ }^{2}$ by filling gaps in measures of local tobacco policies and providing protocols to measure multiple tobacco products in the social, communications and policy environment.

The rapid evolution of the media environment itself represents a challenge. Whereas most measures of tobacco-related media exposure focus on television, media or the internet in general, it is likely that opportunities for exposure to tobacco products and messages via social media sites, online apps and interactive games will continue to increase. Measures will be needed to assess tobacco exposure in these media as well. As the use and ownership of digital devices become more pervasive across all age groups, we recommend the development of new and creative methods to estimate the influence of tobacco-related digital messages and imagery on youth and adults.

\section{International implications}

Although these measures were selected for the US context, most are also relevant to International Tobacco Surveys. However, the measures will need to be customised to reflect policy variations across countries (eg, requirements for plain packaging or graphical warning labels, variations in youth access policies and differences in the content of local antitobacco media campaigns). The use of similar measures across countries could elucidate the interactions among policies, media strategies and cultural norms in reducing tobacco use. Applying these measures across counties could also provide evidence for common risk and protective 
factors across populations that experience tobacco-related health disparities, such as indigenous populations and other marginalised groups in various countries.

\section{Limitations}

Many of the available measures that are relevant to multiple tobacco products came from the PATH Study but other surveys were considered as well. Many measures are based on sound principles but do not yet have published studies documenting their reliability and validity. Such studies, as they are published, will increase the evidence base for the items considered for the PhenX Toolkit.

The community outreach process generated 13 responses out of the thousands of researchers who might use these measures. Response from a wider cross-section of the TRR community may have elicited different feedback, which may have resulted in different content in the Toolkit.

\section{CONCLUSION}

The PhenX Environment WG identified and documented 13 measures of environmental constructs that can influence tobacco product use. Consistent use of these measures across studies should facilitate data harmonisation, reproducibility of research and comparisons across population groups. Continued efforts are needed to encourage tobacco researchers to use PhenX measures, including mentioning PhenX in requests for grant applications and publication of articles such as the ones in this issue.

\section{What this paper adds}

- Environmental factors such as social norms, industry advertising, and state and local policies are well known to influence the initiation and maintenance of the use of tobacco products, and thus, are highly relevant to regulatory considerations.

- The continuous proliferation of novel tobacco products as advertised and marketed across a variety of platforms introduces challenges to scientists seeking to support evidence-based regulatory decision-making because suitable measures may not be readily available or widely known.

- The Environment Working Group filled previously noted gaps by providing, for the first time, consensus recommendations to investigators in the field regarding best methods to assess exposure to multiple social, communications and policy (including local tobacco policy) environmental influences on tobacco product use in youth and adults.

- As tobacco regulatory science evolves, it will be critical that investigators consistently use valid and comprehensive measures of multiple sources of environmental influence on tobacco product use.

Acknowledgements We gratefully acknowledge the contributions of the anonymous members of the scientific community who commented on the measures proposed for inclusion. We also acknowledge the contributions of Deborah Maiese, who led the recruitment of Working Group members and the orientation to the PhenX process, and the contributions of the RTI editorial team, including Debbie Bond, Loraine Monroe and Amy Morrow, for providing expert editorial review of the manuscript.

Collaborators The following aremembers of the PhenX TobaccoRegulatory Research (TRR) Environment Working Group: Co-Chairs Frank J Chaloupka, Universityof Illinois at Chicago and Donna Vallone, Truth Initiative; Working GroupMembers Jamie F Chriqui,University of Illinois at Chicago, Joanna E Cohen,
Johns Hopkins BloombergSchool of Public Health, Jim F Thrasher, University of South Carolina, andJennifer B Unger, University of Southern California; Special Consultant MichaelP Eriksen, GeorgiaState University; TRR Panel Liaisons Andrew Hyland, Roswell Park ComprehensiveCancer Center and Gordon Willis, National Cancer Institute; NIH Liaison RachelGrana Mayne, National Cancer Institute; NIH Project Coordinator, Kay L Wanke,NIH Office of Disease Prevention; and the PhenX team from RTI Internationalincluding Tabitha P Hendershot, TRR RTI Project Lead, Destiny S Nettles, WG Supervisor, and RebeccaGeisler, WG manager.

Contributors GES, TPH, and KLWled the conceptualisation of the manuscript and the coordination of the writingteam. JBU and GES drafted the scientific content of the manuscript, and TPH andDSN drafted the Methods and Results sections of the manuscript. FJC, DV, and JFT reviewed the draft andprovided substantive revisions. Enver Holder-Hayes, FDA Center for Tobacco Productsand RGM reviewed the draft and provided comments. T he Co-Chairs and Members of the PhenX TRR Environment Working Group identified andproposed preliminary measures and voted on final measures included in the PhenXTRR Environment Specialty Collection. PhenX TRR Panel members AH and GWensured that the Working Group process maintained fidelity with overall projectgoals. Federal Agency Liaisons RG and EH-H ensured project consistency withagency goals and priorities. NIH Project Coordinator KW proposed the PhenX TRRinitiative and contributed to its execution and completion. The PhenX team coordinated and facilitatedthe Working Group process, including project oversight and leadership (TPH),supervisory management (DSN), and project management (RG). Erin M Ramos, National Human Genome Research Institute, is the PhenX NIH Program Official, provided project guidance and fundingcoordination. Carol M Hamilton, RTIInternational, is the PhenX Principal Investigator, and provided project guidance andsupervision.

Funding Research reported in this publication was supported by grant number U41HG007050 and U41HG007050-01S1 from the NIH National Human Genome Research Institute (NHGRI) and FDA Center for Tobacco Products (CTP).

Disclaimer The content is solely the responsibility of the authors and does not necessarily represent the official views of the NIH or the Food and Drug Administration.

Competing interests None declared.

Patient consent Not required.

Provenance and peer review Not commissioned; externally peer reviewed.

ORCID iD

Jennifer B Unger http://orcid.org/0000-0001-9064-6603

\section{REFERENCES}

1 Giovino GA, Biener L, Hartman AM, et al. Monitoring the tobacco use epidemic I. Overview: optimizing measurement to facilitate change. Prev Med 2009;48(1 Suppl):S4-10.

2 Farrelly MC. Monitoring the tobacco use epidemic V: the environment: factors that influence tobacco use. Prev Med 2009:48:S35-S43.

3 Centers for Disease Control and Prevention. Best practices for comprehensive tobacco control programs - 2014. Atlanta, GA: U.S. Department of Health and Human Services, National Center for Chronic Disease Prevention and Health Promotion, Office on Smoking and Health, 2014.

4 Xu X, Alexander RL, Simpson SA, et al. A cost-effectiveness analysis of the first federally funded antismoking campaign. Am J Prev Med 2015;48:318-25

5 Niederdeppe J. Conceptual, Empirical, and Practical Issues in Developing Valid Measures of Public Communication Campaign Exposure. Commun Methods Meas 2014:8:138-61.

6 Davis KC, Nonnemaker JM, Farrelly MC. Association between national smoking prevention campaigns and perceived smoking prevalence among youth in the United States. J Adolesc Health 2007;41:430-6.

7 Farrelly MC, Healton CG, Davis KC, et al. Getting to the truth: evaluating national tobacco countermarketing campaigns. Am J Public Health 2002;92:901-7.

8 McAfee T, Davis KC, Alexander RL, et al. Effect of the first federally funded US antismoking national media campaign. Lancet 2013:382:2003-11.

9 Vallone DM, Duke JC, Cullen J, et al. Evaluation of EX: a national mass media smoking cessation campaign. Am J Public Health 2011;101:302-9.

10 Sly DF, Heald GR, Ray S. The Florida "truth" anti-tobacco media evaluation: design, first year results, and implications for planning future state media evaluations. Tob Control 2001;10:9-15.

11 Niederdeppe J. Assessing the validity of confirmed AD recall measures for public health communication campaign evaluation. J Health Commun 2005;10:635-50.

12 Thrasher JF, Huang L, Pérez-Hernández R, et al. Evaluation of a social marketing campaign to support Mexico City's comprehensive smoke-free law. Am J Public Health 2011;101:328-35.

13 McNeill A, Gravely S, Hitchman SC, et al. Tobacco packaging design for reducing tobacco use. Cochrane Database Syst Rev 2017;4:CD011244. 
14 Dunlop SM, Cotter T, Perez D. When your smoking is not just about you: antismoking advertising, interpersonal pressure, and quitting outcomes. J Health Commun 2014;19:41-56.

15 Dunlop SM, Wakefield M, Kashima Y. The contribution of antismoking advertising to quitting: intra- and interpersonal processes. J Health Commun 2008;13:250-66.

16 Durkin S, Wakefield M. Maximizing the impact of emotive antitobacco advertising: effects of interpersonal discussion and program placement. Soc Mar Q 2006;12:3-14.

17 Hafstad A, Aaro LE. Activating interpersonal influence through provocative appeals: evaluation of a mass media-based antismoking campaign targeting adolescents. Health Commun 1997:9:253-72.

18 van den Putte B, Yzer M, Southwell BG, et al. Interpersonal communication as an indirect pathway for the effect of antismoking media content on smoking cessation. $J$ Health Commun 2011;16:470-85.

19 Thrasher JF, Abad-Vivero EN, Huang L, et al. Interpersonal communication about pictorial health warnings on cigarette packages: policy-related influences and relationships with smoking cessation attempts. Soc Sci Med 2016;164:141-9.

20 FDA. The Real Cost: Research + Evaluation. 2017 www.fda.gov/downloads/ TobaccoProducts/PublicHealthEducation/PublicEducationCampaigns/ TheRealCostCampaign/UCM384308.pdf.

21 Babb S, McNeil C, Kruger J, et al. Secondhand smoke and smoking restrictions in casinos: a review of the evidence. Tob Control 2015;24:11-17.

22 Ferketich AK, Lugo A, La Vecchia C, et al. Relation between national-level tobacco control policies and individual-level voluntary home smoking bans in Europe. Tob Control 2016;25:60-5.

23 Hahn EJ, Rayens MK, Adkins S, et al. A controlled community-based trial to promote smoke-free policy in rural communities. J Rural Health 2015;31:76-88.

24 Kadowaki J, Vuolo M, Kelly BC. A review of the current geographic distribution of and debate surrounding electronic cigarette clean air regulations in the United States. Health Place 2015;31:75-82.

25 Klein EG, Hood NE. The smoking ban next door: do hospitality businesses in border areas have reduced sales after a statewide smoke-free policy? Health Policy 2015;119:44-9.

26 Fagan $\mathrm{P}$, Moolchan ET, Lawrence $\mathrm{D}$, et al. Identifying health disparities across the tobacco continuum. Addiction 2007;102(Suppl 2):5-29.

27 Hartwell G, Thomas S, Egan M, et al. E-cigarettes and equity: a systematic review of differences in awareness and use between sociodemographic groups. Tob Control 2017;26:1-7.

28 Lee JG, Henriksen L, Rose SW, et al. A systematic review of neighborhood disparities in point-of-sale tobacco marketing. Am J Public Health 2015; 105:e8-18

29 Asumda F, Jordan L. Minority youth access to tobacco: a neighborhood analysis of underage tobacco sales. Health Place 2009:15:140-7.

30 Borrell LN, Kiefe Cl, Diez-Roux AV, et al. Racial discrimination, racial/ ethnic segregation, and health behaviors in the CARDIA study. Ethn Health 2013;18:227-43.

31 Gonzalez M, Sanders-Jackson A, Song AV, et al. Strong smoke-free law coverage in the United States by race/ethnicity: 2000-2009. Am J Public Health 2013;103:e62-6.

32 Siahpush M, Jones PR, Singh GK, et al. Association of availability of tobacco products with socio-economic and racial/ethnic characteristics of neighbourhoods. Public Health 2010;124:525-9.

33 Widome R, Brock B, Klein EG, et al. Smokeless tobacco advertising at the point of sale: prevalence, placement, and demographic correlates. Nicotine Tob Res 2012:14:217-23.

34 Widome $R$, Brock $B$, Noble $P$, et al. The relationship of neighborhood demographic characteristics to point-of-sale tobacco advertising and marketing. Ethn Health 2013;18:136-51.

35 Zhao X, Strasser A, Cappella JN, et al. A measure of perceived argument strength: reliability and validity. Commun Methods Meas 2011;5:48-75.

36 Davis KC, Nonnemaker J, Duke J, et al. Perceived effectiveness of cessation advertisements: the importance of audience reactions and practical implications for media campaign planning. Health Commun 2013;28:461-72.

37 Tynan MA, Polansky JR, Titus K, et al. Tobacco use in top-grossing movies - United States, 2010-2016. MMWR Morb Mortal Wkly Rep 2017;66:681-6.

38 WHO. Smoke-free movies: from evidence to action. Third edition. $2015 \mathrm{http}: / / a p p s$. who.int/iris/bitstream/10665/190165/1/9789241509596_eng.pdf?ua=1.

39 Sargent JD, Beach ML, Dalton MA, et al. Effect of seeing tobacco use in films on trying smoking among adolescents: cross sectional study. BMJ 2001;323:1394-7.

40 Sargent JD, Worth KA, Beach M, et al. Population-based assessment of exposure to risk behaviors in motion pictures. Commun Methods Meas 2008;2:134-51.
41 Henriksen L, Schleicher NC, Feighery EC, et al. A longitudinal study of exposure to retail cigarette advertising and smoking initiation. Pediatrics 2010;126:232-8.

42 Fishbein M, Cappella JN. The role of theory in developing effective health communications. J Commun 2006;56:S1-S17.

43 Hitchman SC, Fong GT, Zanna MP, et al. Socioeconomic status and smokers' number of smoking friends: findings from the International Tobacco Control (ITC) Four Country Survey. Drug Alcohol Depend 2014;143:158-66.

44 Hitchman SC, Fong GT, Zanna MP, et al. The relation between number of smoking friends, and quit intentions, attempts, and success: findings from the International Tobacco Control (ITC) Four Country Survey. Psychol Addict Behav 2014;28:1144-52.

45 NIH \& FDA. Population Assessment of Tobacco and Health (PATH) Study: Final Adult Baseline (Wave 1) Questionnaire. Bethesda, MD: NIH, National Institute on Drug Abuse, U.S. Department of Health and Human Services, FDA, Center for Tobacco Products, 2013.

46 Chapman S, Freeman B. Markers of the denormalisation of smoking and the tobacco industry. Tob Control 2008;17:25-31.

47 Mead EL, Rimal RN, Ferrence R, et al. Understanding the sources of normative influence on behavior: the example of tobacco. Soc Sci Med 2014;115:139-43.

48 Rimal RN, Real K. Understanding the influence of perceived norms on behaviors. Communication Theory 2003;13:184-203.

49 ITC Policy Evaluation Project. International Tobacco Control (ITC) 4-Country Survey: ITC Policy Evaluation Project, 2011. Item 365.

50 NIH \& FDA. Population Assessment of Tobacco and Health (PATH) Study, Wave 1 Adult Extended Interview 7.5. Items AX0066, AX0071, AX0007. Ann Arbor, MI: NIH, National Institute on Drug Abuse, U.S. Department of Health and Human Services, FDA, Center for Tobacco Products, 2012.

51 NIH \& FDA. Population Assessment of Tobacco and Health (PATH) Study, Wave 1 Youth Extended Interview 7.5. Item YX0671. Ann Arbor, MI: NIH, National Institute on Drug Abuse, U.S. Department of Health and Human Services, FDA, Center for Tobacco Products, 2013

52 Chassin L, Presson CC, Sherman SJ, et al. Changes in peer and parent influence during adolescence: longitudinal versus cross-sectional perspectives on smoking initiation. Dev Psychol 1986:22:327-34.

53 Powell LM, Tauras JA, Ross H. The importance of peer effects, cigarette prices and tobacco control policies for youth smoking behavior. J Health Econ 2005;24:950-68.

54 Centers for Disease Control and Prevention (CDC). State smoking restrictions for private-sector worksites, restaurants, and bars--United States, 1998 and 2004. MMWR Morb Mortal Wkly Rep 2005;54:649-53.

55 Centers for Disease Control and Prevention (CDC). State smoking restrictions for private-sector worksites, restaurants, and bars--United States, 2004 and 2007. MMWR Morb Mortal Wkly Rep 2008;57:549-52.

56 Centers for Disease Control and Prevention (CDC). State preemption of local tobacco control policies restricting smoking, advertising, and youth access--United States, 2000-2010. MMWR Morb Mortal Wkly Rep 2011:60:1124-7.

57 Centers for Disease Control and Prevention (CDC). State smoke-free laws for worksites, restaurants, and bars--United States, 2000-2010. MMWR Morb Mortal Wkly Rep 2011;60:472-5.

58 Centers for Disease Control and Prevention. Comprehensive smoke-free laws-50 largest U.S. cities, 2000 and 2012. Morb Mortal Wkly Rep 2012;61:914-7.

59 Marynak K, Holmes CB, King BA, et al. State laws prohibiting sales to minors and indoor use of electronic nicotine delivery systems--United States, November 2014 MMWR Morb Mortal Wkly Rep 2014;63:1145-50.

60 Singleterry J, Jump Z, Lancet E, et al. State Medicaid coverage for tobacco cessation treatments and barriers to coverage - United States, 2008-2014. MMWR Morb Mortal Wkly Rep 2014:63:264-9.

61 McDaniel PA, Malone RE. Businesses' voluntary pro-health tobacco policies: a review and research agenda. Tob Control 2012:21:66-72.

62 Centers for Disease Control and Prevention. National Adult Tobacco Survey Questionnaire. Questions 77-84. Atlanta, GA: National Center for Chronic Disease Promotion and Health Prevention, Office on Smoking and Health 2009-2010.

63 King BA, Homa DM, Dube SR, et al. Exposure to secondhand smoke and attitudes toward smoke-free workplaces among employed U.S. adults: findings from the National Adult Tobacco Survey. Nicotine Tob Res 2014;16:1307-18.

64 University of Michigan Institute for Social Research, Survey Research Center. Monitoring the future: a continuing study of American youth. Part B, Questions 3-7. Ann Arbor, MI: U.S. Department of Health and Human Services, NIH, National Institute on Drug Abuse, 2010.

65 Johnston LD, O'malley PM, Terry-McElrath YM. Methods, locations, and ease of cigarette access for American youth, 1997-2002. Am J Prev Med 2004;27:267-76. 